\title{
Electronic Structure of Lanthanum Hydrides with Switchable Optical Properties
}

\author{
K. K. Ng ${ }^{1,2}$, F. C. Zhang ${ }^{1,2}$, V. I. Anisimov ${ }^{2,3}$, and T. M. Rice ${ }^{2}$ \\ ${ }^{1}$ Department of Physics, University of Cincinnati, Cincinnati, Ohio 45221 \\ ${ }^{2}$ Theoretische Physik, ETH-Hönggerberg, 8093 Zürich, Switzerland \\ ${ }^{3}$ Institute of Metal Physics, Russian Academy of Sciences, 620219, Ekaterinburg, GSP-170, Russia
}

(October 22, 2018)

\begin{abstract}
Recent dramatic changes in the optical properties of $\mathrm{LaH}_{2+x}$ and $\mathrm{YH}_{2+x}$ films discovered by Huiberts et al. suggest their electronic structure is described best by a local model. Electron correlation is important in $H^{-}$-centers and in explaining the transparent insulating behavior of $\mathrm{LaH}_{3}$. The metal-insulator transition at $x \sim 0.8$ takes place in a band of highly localized states centered on the $H$-vacancies in the $\mathrm{LaH}_{3}$ structure.
\end{abstract}

Recently Huiberts et. al. [1] reported dramatic changes in the optical properties of lanthanum and yttrium hydride films with changing hydrogen content, e.g. films that switch from a shiny mirror to a yellow transparent window in a time of order seconds. Although many metal-insulator transitions are known, it is very unusual and potentially of technological importance, that the transition leads to spectacular effects in the visible. Huiberts et. al. [1] point out that the electronic structure that underlies this behavior is poorly understood - indeed standard LDA-calculations do not predict a metalinsulator transition at all [2,3. In this letter we examine the role of electron correlation in these hydrides and argue it justifies a local description that derives from an ionic starting point.

The structural changes are especially small in the $\mathrm{LaH}_{2+x}$ films. The $\mathrm{La}$-atoms always form a fcc lattice with two $H$-atoms always occupying tetrahedrally coordinated sites. As $x$ changes from 0 to 1 , the octahedrally coordinated site goes from empty to fully occupied and a good metal $\left(\mathrm{LaH}_{2}\right)$ evolves to a transparent insulator $\left(\mathrm{LaH}_{3}\right)$. The metal-insulator transition in the d.c. conductivity [1] occurs at an intermediate concentration, $x \sim 0.8$, far removed from a lightly doped semiconductor. This in turn suggests highly localized states associated with $\mathrm{H}$-vacancies in $\mathrm{LaH}_{3}$. The key challenge is to understand why the structural changes with changing hydrogen content are remarkably small, but the changes in spectral properties are dramatic at energy scales up to a visible and secondly the origin of the highly localized states at intermediate concentrations. We shall address these issues in turn.

We have performed self-consistent LDA calculations on $\mathrm{LaH}_{2}$ and $\mathrm{LaH}_{3}$. In agreement with previous results [2] there are two sets of states - low energy primarily $1 s$ $H$-states and higher energy states of mainly $5 d-L a$ character. These results suggest assigning a formal valence $\mathrm{H}^{-}$. In $\mathrm{LaH}_{2}$ the $\mathrm{H}^{-}$-bands are filled leaving $1 \mathrm{el} / \mathrm{f}$.u. in the $5 d-L a$ conduction bands leading to metallic behaviour [2]. In $\mathrm{LaH}_{3}$ the $\mathrm{H}^{-}$-bands can hold all $6 \mathrm{el} / \mathrm{f}$.u. but in the LDA calculations there is overlap between the $H^{-}$and $5 d-L a$ bands leading to a semimetal rather than the observed transparent insulator.

The LDA calculations give an interesting insight why atomic $H$ is easily incorporated in this structure. The net charge change between $\mathrm{LaH}_{2}$ and $\mathrm{LaH}_{3}$ is found to be remarkably small. The charge at the octahedral site (within a sphere of 2.533 atomic unit (a.u.) radius) in $\mathrm{LaH}_{3}$ is $1.588 \mathrm{el}$., while the charge in an empty sphere of the same radius in $\mathrm{LaH}_{2}$ is $0.568 \mathrm{el}$., so to a very good accuracy, $\mathrm{LaH}_{2+x}$ can be interpreted as neutral $\mathrm{H}$-atoms moving into or out of the octahedral interstitial sites, with negligible changes in the lattice structure. This charge distribution of $\mathrm{LaH}_{3}$ is not very different in an ionic picture due to a large radius $(\sim 2 a . u$. $)$ of the outer electron in the bound state of the $H^{-}$-ion.

The $H^{-}$-ion is a difficult case for LDA and a careful treatment of the correlation between the two electrons is required [4] 6] to obtain the binding energy $(\simeq 0.7 \mathrm{eV})$. This led us to examine the effect of correlations on the width of the $H^{-}$-bands. We start by considering the hopping integral $t(d)$ for an electron between $H$-atoms with separation $d$ (in a.u.), ignoring for the moment the $\mathrm{La}$-ions. This can be obtained at once from the lowest energy levels of the $\mathrm{H}_{2}^{-}$-ion with inter-proton distance, d. $H_{2}^{-}$is a bound negative ion for $d>3$ - the range of interest here.

Let $\psi_{i}(1,2)$ be the groundstate wavefunction of two electrons in $H^{-}$, and $\phi_{i}(1)$ be the groundstate of hydrogen atom. The three electron states of the $\mathrm{H}_{2}^{-}$ with odd(-) and even(+) parities may be constructed using the single site (i and $\mathrm{j}$ ) states: $\Psi_{ \pm}=\Phi_{i, j} \pm$ $\Phi_{j, i}$, where $\Phi_{i, j}=A\left[\psi_{i}(1,2) \phi_{j}(3)\right]$, and $A$ is an antisymmetrizer. The corresponding energies are given by $E_{ \pm}=\left\langle\Psi_{ \pm}|h| \Psi_{ \pm}\right\rangle /\left\langle\Psi_{ \pm} \mid \Psi_{ \pm}\right\rangle$, where $h$ is the Hamiltonian for the $H_{2}^{-}$-system, including the kinetic and Coulomb energies [7]. We choose a variational state of Chandrasekhar 河 for $H^{-}$:

$$
\psi(1,2)=\left(e^{-a r_{1}-b r_{2}}+e^{-a r_{2}-b r_{1}}\right)\left(1+c\left|\vec{r}_{1}-\vec{r}_{2}\right|\right) \chi,
$$


where $r$ are the electron radial coordinates, $\chi$ is the spinor for a singlet, and $a=1.075, b=0.478, c=0.312$ in a.u.. This simple wavefunction describes two intraatomic correlated electrons well, and its energy is very close to the best estimate of Hylleraas [6] involving 24 variational parameters. The states for $\mathrm{H}_{2}^{-}$thus constructed become exact in the limit $d \gg 1$. The effective hopping integral for an electron between two $H$-sites can be obtained, $t(d)=\left(E_{-}-E_{+}\right) / 2$. At $d=4$, the groundstate energy estimated in this method is $E_{-}=-1.032$ a.u., very close to the best available result of -1.034 a.u. for $H_{2}^{-}$using more complicated variational method [8]. This suggests that the groundstate of $H_{2}^{-}$at these separations is very well described by a linear combination of $H^{-}$and $H$-states, and justifies our approach.

The two shortest inter-hydrogen distances in $\mathrm{LaH}_{3}$ are those between n.n. tetra- and octa-sites (denoted as $H_{\text {tet }}$ and $\left.H_{\text {oct }}\right)$ and n.n. tetra-sites which denote as $t_{2}$ and $t_{1}$ respectively. These distances are $\sqrt{3} a_{0} / 4$ and $a_{0} / 2$ ( $a_{0}$ : the lattice constant) respectively (4.58 and 5.29 a.u.) giving values $t_{2}=-0.74 \mathrm{eV}$ and $t_{1}=-0.54 \mathrm{eV}$. The $H^{-}$bands in tight binding representation are the eigenstates of the Hamiltonian

$$
\mathcal{H}_{\mathrm{tb}}=\sum_{\vec{k}} c^{\dagger}(\vec{k}) M(\vec{k}) c(\vec{k})
$$

where $c^{\dagger}(\vec{k})$ is a 3 -component vector, representing the 3 $H$-states in a unit cell, and

$$
M(\vec{k})=\left(\begin{array}{ccc}
\varepsilon_{t} & t_{1} \alpha(\vec{k}) & t_{2} \beta(\vec{k}) \\
t_{1} \alpha(\vec{k}) & \varepsilon_{t} & t_{2} \beta^{*}(\vec{k}) \\
t_{2} \beta^{*}(\vec{k}) & t_{2} \beta(\vec{k}) & \varepsilon_{o}
\end{array}\right) .
$$

with

$$
\begin{gathered}
\alpha(\vec{k})=\sum_{\tau=x, y, z} 2 \cos \left(k_{\tau} / 2\right), \\
\beta(\vec{k})=\sum_{\sigma_{1}, \sigma_{2}= \pm 1} e^{i\left(\sigma_{1} k_{x}+\sigma_{2} k_{y}+\sigma_{1} \sigma_{2} k_{z}\right) / 4} .
\end{gathered}
$$

Note we now use the lattice parameter $a_{0}(5.60 \AA)$ as the length unit. The atomic energy levels at $H_{\text {tet }}$ and $H_{\text {oct }}$ ( $\varepsilon_{t}$ and $\varepsilon_{0}$ ) we estimate using the LDA calculations as follows. At the high symmetry $\Gamma$ point, one can identify in the LDA a 4 by 4 matrix corresponding to the three $H$ and one $L a-6$ s states in a unit cell of the fcc lattice. We can map this matrix onto a 3 by 3 submatrix describing the effective three $H$-bands with the same three lower eigenenergies. The highest energy band, representing the La-6s state, is pushed well above the bottom of the $L a-$ $5 d$ conduction band due to the strong hybridization, and can be projected out. Incorporating the binding state energy of a single $H^{-}$-ion, which is $0.7 \mathrm{eV}$, we obtain $\varepsilon_{t}=-3.9 \mathrm{eV}$, and $\varepsilon_{0}=-3.3 \mathrm{eV}$.
In Fig. 1a the resulting energy bands are plotted. We find a small gap, which is not sufficient for a transparent insulator. However we have neglected the $L a^{3+}$-ions in estimating $\left(t_{1}, t_{2}\right)$. In an ionic picture the $L a^{3+}$-ions generate a crystal field at the $H$-sites whose effect can be estimated as follows. Let the interaction between an electron in $H_{2}^{-}$and the other ions be $h^{\prime}=-\sum_{\vec{R}} Z(\vec{R}) e^{2} /|\vec{r}-\vec{R}|$, with $Z=3$ for $L a^{3+}$, and $\mathrm{Z}=-1$ for $H^{-}$-ions, and $\vec{R}$ the ion position. The energies for $\mathrm{H}_{2}^{-}$of two $H_{\text {tet }}$ are modified, due to the crystal field, to $E_{ \pm}^{\prime}$, which is obtained by adding $h^{\prime}$ to $h$. The reduction of $t_{1}$ due to the crystal field is found to be $\delta t_{1}=0.20 \mathrm{eV}$, where the contribution from within a cubic unit cell is $0.08 \mathrm{eV}$. The estimate for $t_{2}$ is more complicated because of the Madelung constants. Assuming the same percentage reduction, we estimate the hoppings in the presence of the crystal field, $\tilde{t}_{1}=-0.34 \mathrm{eV}, \tilde{t}_{2}=-0.47 \mathrm{eV}$. The estimate is based on an ideal ionic approach, and neglects the screening effect, so that the actual reduction of $t$ 's are surely smaller. With the new values $\left(\tilde{t}_{1}, \tilde{t}_{2}\right)$, we obtain strongly narrowed $H^{-}$bands (see Fig. 1) and a transparent insulating ground state. This idealized ionic approach overestimates the narrowing and neglects the effects of hybridization on the hopping integrals. These however are weak at the top of the $H^{-}$-bands and are mainly important only at energies well below the Fermi energy.

Turning to the metal-insulator transition in $\mathrm{LaH}_{2+x}$ as $x$ increases, we can view it starting either at the metallic $(x=0)$ or insulating $(x=1)$ endpoints. In the former case, the introduction of a neutral $H$-atom into a $\mathrm{H}_{\text {oct }^{-}}$ site creates a $s=1 / 2$ magnetic impurity, which couples to the conduction $5 d-L a$ electron spins. The effective Hamiltonian is a Kondo model,

$$
\mathcal{H}_{\mathrm{eff}}=\sum_{\vec{k}, \sigma} \varepsilon(\vec{k}) d_{\vec{k}, \sigma}^{\dagger} d_{\vec{k}, \sigma}+J \sum_{i} \vec{S}_{i} \vec{s}_{i}
$$

where $i$ runs over all the occupied $H_{\text {oct }}$, and $\vec{S}$ and $\vec{s}$ are the electron spins of the neutral $H$ and of the conduction electron states. The latter represent linear superposition of the $L a-5 d-e_{g}$ states around three degenerate $X_{1}$ points, which is of $s-$ wave symmetry and can couple to the $1 s-H$ state. The antiferromagnetic coupling $J \sim 0.7$ $\mathrm{eV}$ in free space, and may be renormalized by a numerical factor in hydrides. In this Kondo description conduction electrons are captured by the neutral $H$-atoms at $H_{\text {oct- }}$ sites to form tightly bound singlets and $\mathrm{LaH}_{3}$ is viewed as a Kondo insulator with a large band gap.

Starting from insulating $\mathrm{LaH}_{3}$, removing neutral $\mathrm{H}$ atoms causes vacancies at octa-sites $\left(H_{\text {oct }}^{V}\right)$ which donate an electron to the conduction band. In a conventional semiconductor such as in the doped $S i$, the impurity state is described by an effective mass theory, and the result is a hydrogen-like bound state with a large effective Bohr radius $a_{B}^{*}$ of order of hundred $\stackrel{\circ}{A}$ due to the light mass and the large dielectric constant. The critical 
impurity concentration $x_{c}$ at which the system becomes metallic is given by Mott criterion $x_{c} \sim\left(l / a_{B}^{*}\right)^{3}$, where $l$ is the interatomic distance. Because of $a_{B}^{*} \gg l, x_{c}$ is very small $(0.1$ percent for $S i)$. The vacancy state in $\mathrm{LaH}_{2+x}$ is very different, however. Experimentally, it is found that the semiconducting states extend to $x=0.25$ for lanthanum hydrides, and to even larger value for yttrium hydrides [1,9]. Below we shall show that the vacancy state in lanthanum hydrides is an octahedral s-like $L a-e_{g}$ state with extremely small size.

The $L a-5 d-e_{g}$ and $H_{o c t}$ electrons hybridize strongly to form bonding (mainly $H$ ) and anitbonding (mainly $e_{g}$ ) states. A vacancy of $H_{\text {oct }}$ breaks the bonds locally and the $e_{g}$ electron becomes locally non-bonding, which has much lower energy than the antibonding $e_{g}$ states away from the vacancy. Therefore, in addition to serving as a positive charge center as in conventional n-type semiconductors, $H_{\text {oct }}^{V}$ creates a potential well for the $e_{g}$ state electron. The latter is non-perturbative, and is responsible to the unusual concentration-dependence of the semiconductor.

Consider a $s$ - symmetric octahedral $e_{g}$ state $(S$-state hereafter) around a $H_{\text {oct }}^{V}$ as shown in Fig. 2. The $H_{\text {oct }}^{V}$ vacancy breaks the bonds on the octahedron and reduces the anitbonding energy of the $S$-state. This $S$ state is very localized because the neighboring octahedral $s$ - state is antibonding and has a much higher energy. We can estimate the depth of the potential well, $V_{0}$, within a perturbation theory upto the second order in $V_{s d \sigma}$, the hopping integral between an atomic $d_{3 z^{2}-r^{2}}$ and its neighboring $H_{\text {oct }}$ with the orbits towards each other. Let $\Delta$ be the atomic energy difference between $L a-5 \mathrm{~d}$ and $H_{\text {oct }}$, then $V_{0}=-6 V_{s d \sigma}^{2} / \Delta$. This is $2 / 3$ of the antibonding energy $9 V_{s d \sigma}^{2} / \Delta$ at the $X_{1}$ point in $\mathrm{LaH}_{3}$. The latter is estimated to be $\sim 6 \mathrm{eV}$ from LDA [10], so that $V_{0} \sim-4 \mathrm{eV}$. We may also compare this $S$ state to the non-bonding $t_{2 g}$ state. The latter is the lowest energy conduction state of $\mathrm{LaH}_{3}$, and would be a starting point for an effective mass theory in conventional semiconductors. The $S$ state has much better kinetic energy as evidenced from LDA for $\mathrm{LaH}_{2}$ [2, 10, 11]. A more detailed calculation based on a tight-binding model of n.n. hopping between $L a$ sites estimates the kinetic energy gain of the $S$ state over the $t_{2 g}$ state is $\sim 0.9 \mathrm{eV}$. This is about to compensate the loss of the antibonding energy of the $S$ state with the neighboring occupied $H_{\text {oct }}$ atoms, which is $1.5 V_{s d \sigma}^{2} / \Delta \sim 1 \mathrm{eV}$. The $S$ state has better Coulomb attraction to the vacancy, because its orbit is towards the central effective charge. Therefore the $S$ state should have lower energy than the $t_{2 g}$ state, and is the lowest energy state for $H_{\text {oct }}$ vacancy. We emphasize that the potential well generated by the $H_{\text {oct }}$ vacancy is short-ranged, different from the long-range Coulomb force. The effective Bohr radius of the impurity state is only half of the lattice constant.

The localized nature of the impurity state in $\mathrm{LaH}_{2+x}$ is consistent with the temperature dependence of the resistivity data. Shinar et. al. [9] reported a phase transition at $T=250 \mathrm{~K}$, above which the resistivity shows variable range hopping, below that temperature, it is coherent state of the defect band. Localized states are a prerequisite for variable range hopping. The coherent state accompanies the onset of superlattice order in the $H_{\text {oct }}^{V}$ vacancies.

The precise nature of the transition in optical properties is not well understood yet. Since the d.c. conductivity is insulating only in the disordered state of $H_{\text {oct }}^{V}$, and is metallic in the ordered one, we argue that the transition should be a disorder driven Anderson transition, instead of a Mott type correlation driven transition.

In conclusion, we have studied the electronic structure of $\mathrm{LaH}_{2+x}$ starting from $\mathrm{H}^{-}$-ionic picture emphasizing the electron correlation. Our calculations give an insulator for $\mathrm{LaH}_{3}$, in agrement with the recent experiments. We propose that a $\mathrm{H}$-vacancy in $\mathrm{LaH}_{3}$ introduces a $s$ wave state centred at the vacant octa-site,with a highly localized form. This state of the vacancy explains why the semiconductor $\mathrm{LaH}_{2+x}$ to be so stable in a wide range of concentration $x(0.8<x<1)$, and is consistent with the experimental observed variable range hopping mechanism for the resisitivity in these materials. The recent experimental activity of Huiberts et. al. [1] has raised many interesting questions in study of metal hydrides. The precise nature and the criterion of the optical transition from transparent to reflecting needs be examined. The role of quantum diffusion of the hydrogen at low temperatures, the order-disorder transitions, and the interactions between octahedral $H$-vacancies, are all interesting open questions.

[1] J.N. Huiberts, R. Griessen, J.H. Rector, R.J. Wijngaarden, J.P. Dekker, D.G. de Groot, and N.J. Koeman, Nature 380, 231 (1996). 1234 (1996).

[2] J.P. Dekker, J. van Ek, A. Lodder, and J.N. Huiberts, J. Phys. Cond. Mat. 5, 4805 (1993).

[3] Y. Wang and M.Y. Chou, Phys. Rev. Lett. 71, 1226 (1993).

[4] H.A. Bethe and E.E. Salpeter, in "Handbuch der Physik Bd. 35", Edited by S. Flugge, Springer-Verlag, (1957).

[5] S. Chandrasekhar, Astrophys. J. 100, 176 (1944).

[6] E.A. Hylleraas and J. Midtdal, Phys. Rev. 103, 829 (1956).

[7] I. Fischer-Hijalmars, Ark. Fys. 16, 33 (1959). Similar method with much less accurate wavefunction for $H^{-}$ was used to study $E_{ \pm}$.

[8] H.S. Taylor and F.E. Harris, J. Chem. Phys. 39, 1012 (1963).

[9] J. Shinar, B. Dehner, R.G. Barnes, and B.J. Beaudry, 
Phys. Rev. Lett. 64, 563 (1990).

[10] M. Gupta and J.P. Burger, Phys. Rev. B22, 6074 (1980).

[11] D.K. Mismer and B.N. Harmon, Phys. Rev. B26, 5644 (1982).
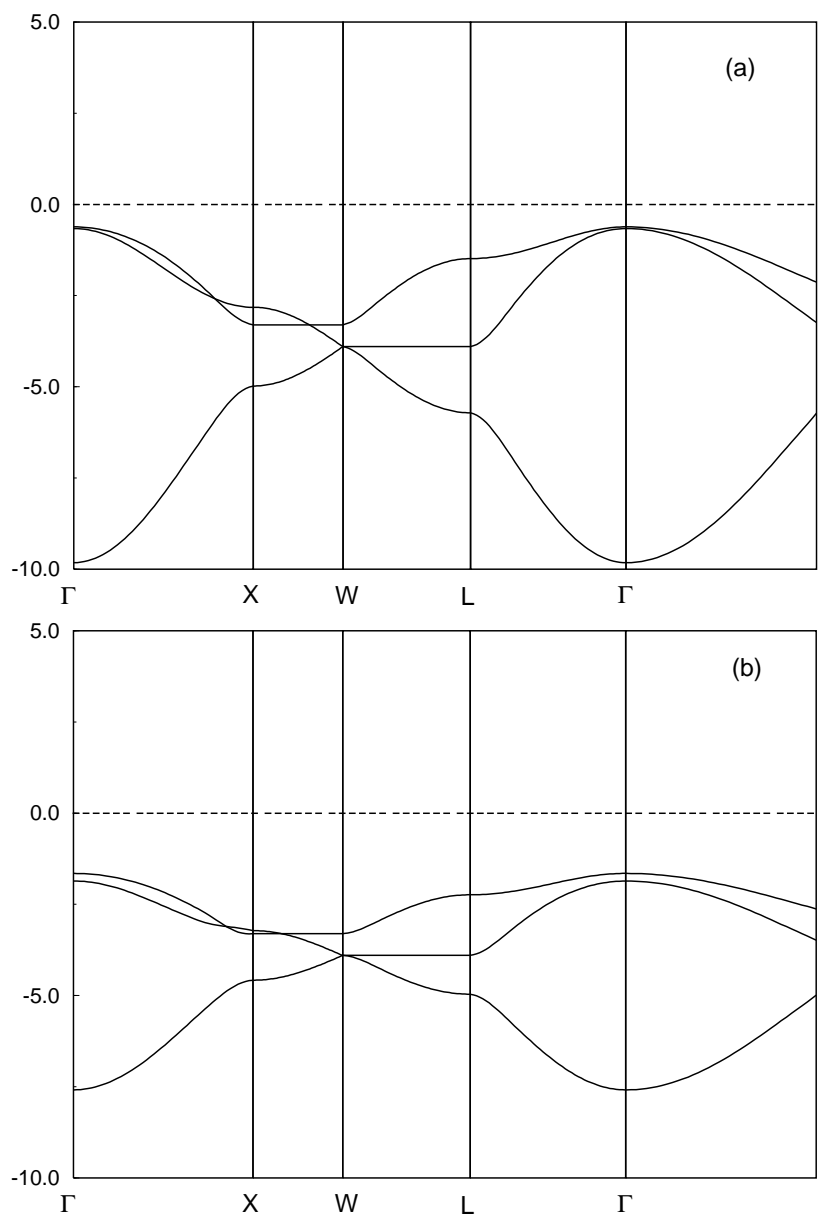

FIG. 1. The valence bands of $\mathrm{LaH}_{3}$ calculated from the single hole hopping in $H^{-}$. The dashed lines indicate the bottom of the conduction bands. a): without crystal fields; b): with the crystal fields

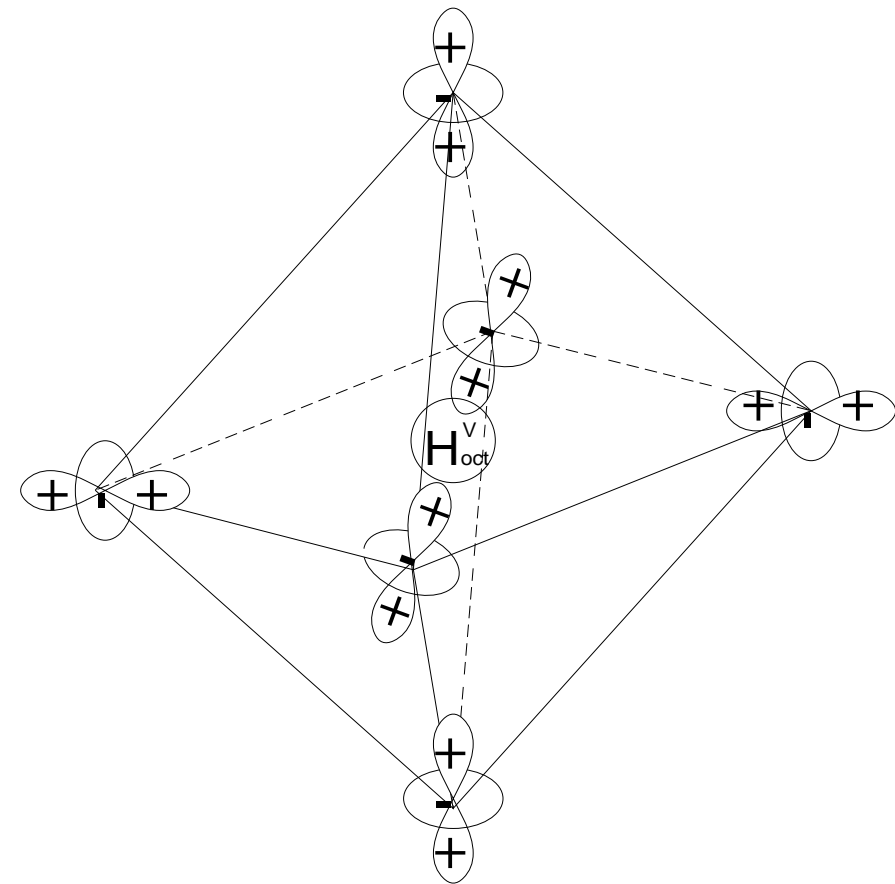

FIG. 2. Diagrammatic illustration of the proposed impurity state in $\mathrm{LaH}_{3}$. The centre circle represents a missing $H$, forming an n-type impurity center. The surrounding orbits represent phases for the local s-like octahedral La-5d- $e_{g}$ state. 